NASA/CR—2005-213576

\title{
Vortex Rings Generated by a Shrouded Hartmann-Sprenger Tube
}

Jack Wilson

QSS Group, Inc., Cleveland, Ohio 
Since its founding, NASA has been dedicated to the advancement of aeronautics and space science. The NASA Scientific and Technical Information (STI) Program Office plays a key part in helping NASA maintain this important role.

The NASA STI Program Office is operated by Langley Research Center, the Lead Center for NASA's scientific and technical information. The NASA STI Program Office provides access to the NASA STI Database, the largest collection of aeronautical and space science STI in the world. The Program Office is also NASA's institutional mechanism for disseminating the results of its research and development activities. These results are published by NASA in the NASA STI Report Series, which includes the following report types:

- TECHNICAL PUBLICATION. Reports of completed research or a major significant phase of research that present the results of NASA programs and include extensive data or theoretical analysis. Includes compilations of significant scientific and technical data and information deemed to be of continuing reference value. NASA's counterpart of peerreviewed formal professional papers but has less stringent limitations on manuscript length and extent of graphic presentations.

- TECHNICAL MEMORANDUM. Scientific and technical findings that are preliminary or of specialized interest, e.g., quick release reports, working papers, and bibliographies that contain minimal annotation. Does not contain extensive analysis.

- CONTRACTOR REPORT. Scientific and technical findings by NASA-sponsored contractors and grantees.
- CONFERENCE PUBLICATION. Collected papers from scientific and technical conferences, symposia, seminars, or other meetings sponsored or cosponsored by NASA.

- SPECIAL PUBLICATION. Scientific, technical, or historical information from NASA programs, projects, and missions, often concerned with subjects having substantial public interest.

- TECHNICAL TRANSLATION. Englishlanguage translations of foreign scientific and technical material pertinent to NASA's mission.

Specialized services that complement the STI Program Office's diverse offerings include creating custom thesauri, building customized databases, organizing and publishing research results ... even providing videos.

For more information about the NASA STI Program Office, see the following:

- Access the NASA STI Program Home Page at http://www.sti.nasa.gov

- E-mail your question via the Internet to help@sti.nasa.gov

- Fax your question to the NASA Access Help Desk at 301-621-0134

- Telephone the NASA Access Help Desk at 301-621-0390

- Write to:

NASA Access Help Desk

NASA Center for AeroSpace Information 7121 Standard Drive

Hanover, MD 21076 
NASA/CR-2005-213576

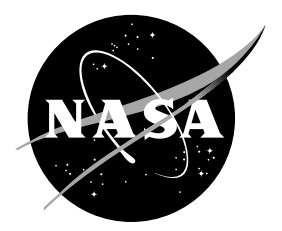

\section{Vortex Rings Generated by a Shrouded Hartmann-Sprenger Tube}

Jack Wilson

QSS Group, Inc., Cleveland, Ohio

Prepared for the

35th Fluid Dynamics Conference and Exhibit

sponsored by the American Institute of Aeronautics and Astronautics

Toronto, Ontario, Canada, June 6-9, 2005

Prepared under Contract NAS3-00145

National Aeronautics and

Space Administration

Glenn Research Center 


\section{Acknowledgments}

The assistance of many people in accomplishing this work is gratefully recognized, in particular Wentworth John, Dan Paxson, and Mark Wernet for the LPIV studies, and Robert Pastel and Kevin Dougherty for assistance with the experiments. Support from the CVCEE project, directed by Leo A. Burkardt, is also acknowledged with gratitude.
Trade names or manufacturers' names are used in this report for identification only. This usage does not constitute an official endorsement, either expressed or implied, by the National Aeronautics and Space Administration.

This work was sponsored by the Low Emissions Alternative

Power Project of the Vehicle Systems Program at the NASA Glenn Research Center.

NASA Center for Aerospace Information 7121 Standard Drive

Hanover, MD 21076
Available from

National Technical Information Service 5285 Port Royal Road Springfield, VA 22100 


\title{
Vortex Rings Generated by a Shrouded Hartmann-Sprenger Tube
}

\author{
Jack Wilson \\ QSS Group, Inc. \\ Cleveland, Ohio 44135
}

The pulsed flow emitted from a shrouded Hartmann-Sprenger tube was sampled with high-frequency pressure transducers and with laser particle imaging velocimetry, and found to consist of a train of vortices. Thrust and mass flow were also monitored using a thrust plate, and orifice, respectively. The tube and shroud lengths were altered to give four different operating frequencies. From the data, the radius, velocity, and circulation of the vortex rings was obtained. Each frequency corresponded to a different length to diameter ratio of the pulse of air leaving the driver shroud. Two of the frequencies had length to diameter ratios below the formation number, and two above. The formation number is the value of length to diameter ratio below which the pulse converts to a vortex ring only, and above which the pulse becomes a vortex ring plus a trailing jet. A modified version of the slug model of vortex ring formation was used to compare the observations with calculated values. Because the flow exit area is an annulus, vorticity is shed at both the inner and outer edge of the jet. This results in a reduced circulation compared with the value calculated from slug theory accounting only for the outer edge. If the value of circulation obtained from laser particle imaging velocimetry is used in the slug model calculation of vortex ring velocity, the agreement is quite good. The vortex ring radius, which does not depend on the circulation, agrees well with predictions from the slug model.

$\begin{array}{ll}a & \text { radius of vortex ring core } \\ D & \text { effective diameter of jet }=\sqrt{D_{s}^{2}-d_{t}^{2}} \\ D_{s} & \text { inner diameter of shroud } \\ d_{t} & \text { outer diameter of resonance tube } \\ E(\varepsilon) & \text { dimensionless energy of vortex ring } \\ f & \text { frequency } \\ I(\varepsilon) & \text { dimensionless impulse of vortex ring } \\ K & \text { circulation } \\ L & \text { length of volume (slug) of air in each pulse } \\ \dot{m}_{\text {jet }} & \text { mass flow rate of jet } \\ m_{\text {pulse }} & \text { mass of air in one pulse } \\ N & \text { formation number } \\ P & \text { vortex ring impulse } \\ R & \text { vortex ring radius } \\ r & \text { distance along radius of vortex ring } \\ T & \text { energy of vortex ring } \\ t & \text { time } \\ T_{j e t} & \text { thrust of jet } \\ U & \text { characteristic jet velocity } \\ u(t) & \text { jet velocity as function of time } \\ U_{l} & \text { average velocity defined in equation (15) } \\ U_{2} & \text { average velocity defined in equation (16) } \\ W & \text { vortex ring velocity } \\ z & \text { distance along axis of vortex ring } \\ \varepsilon & \text { ratio } a / R \\ \Gamma & \text { ratio of ring to slug circulation }\end{array}$




$\begin{array}{ll}\rho & \text { density of air } \\ \tau & \text { duration of one pulse }(1 / f) \\ \omega & \text { vorticity }\end{array}$

\section{Introduction}

In an experiment to study pulsed ejector thrust augmentation, a shrouded Hartmann-Sprenger tube was used to create pulsating flow. ${ }^{1}$ A Hartmann-Sprenger tube is a device which, without any moving parts, transforms a steady flow into a pulsating flow. By shrouding the device, the flow is channeled into an axially directed pulsed jet, which is convenient for ejector studies. This work was performed to establish the properties of this flow.

It is well-known that an impulsively started flow produces a vortex ring (Elder and deHaas, ${ }^{2}$ Das et al. ${ }^{3}$ ). Most studies of this phenomenon have used a single pulse of water propelled into a tank of water by a piston. The velocity and size of the vortex ring can be calculated from the so-called slug model, given the circulation of the flow. The circulation is produced at the outer edge of the flow, and is of the order of $U L / 2$. If the orifice producing the flow is an annulus, rather than a circle, one could argue that a quantity of circulation of the order of $U L / 2$ is produced at the outer diameter, and a quantity of equal magnitude, but opposite sign, is produced at the inner diameter. These should cancel each other, so that no vortex ring will result. The exit geometry of the shrouded Hartmann-Sprenger tube is an annulus, so it is of interest to see whether the train of pulses emerging from it constitutes vortex rings, and if so, whether their velocity and size can be calculated from the slug model, which is intended for a single circular pulse.

In this study, the flow exiting a shrouded Hartmann-Sprenger tube was probed with high-frequency pressure transducers, and found to be a train of vortex rings. The probes located the radius of the ring cores, and were also used to measure the ring velocities, as well as the velocity of the flow at the exit plane of the Hartmann-Sprenger tube. In addition, laser particle imaging velocimetry was used to map the ring velocity and vorticity distribution. The measurements are compared with calculations from the slug model. Modifications to the slug model were found to be necessary in order to achieve good agreement with the data.

\section{Summary of the Slug Model}

In the slug model, as shown in figure 1, the pulsed jet is assumed to consist of repeated slugs of fluid, each of length $L$, with constant velocity $U$, and density $\rho$, issuing from a tube of diameter $D$, as would be produced by a piston impulsively started with velocity $U$ and stopping after a stroke $L$. Initially, the model will be in the usual form, i.e., for a circular jet orifice, not an annulus. According to Gharib, Rambod, and Shariff, ${ }^{4}$ the slugs will transform entirely into vortex rings, provided $L / D \leq 4$. Above a value of $L / D=4$, vortex rings will form with circulation, and size, equal to that produced at $L / D=4$, followed by a trailing jet containing the rest of the circulation. The maximum value of $L / D$ which still produces only a vortex ring with no trailing jet is called the formation number, $N$. Each vortex ring will have a radius $R$, and a core of "radius" $a$. Strictly speaking, the core is not a circle, therefore does not have a radius. However, it is approximately circular, and what is meant by $a$, is the square root of the core area divided by $\pi$. The value of $\varepsilon=a / R$, is small at small values of $L / D$, and increases monotonically up to a value of about 0.53 at the cut-off point.

Given the properties of the jet, Linden and Turner ${ }^{5}$ have shown that the properties of the vortex, i.e., $R, a$, and $W$, are uniquely determined as a function of the value of $L / D$ for the jet. To demonstrate this, they balanced the jet circulation, impulse and energy with those for the ring, taking the ring values from the work of Norbury. ${ }^{6}$ Then with values for the jet in the middle of the equations below, and values for the vortex ring on the right hand side, the balance equations become

Circulation:

Impulse:

Energy:

$$
K_{\text {slug }}=U L / 2 \quad K_{\text {ring }}=\Gamma K_{\text {slug }}
$$

$$
P=\pi \rho U D^{2} L / 4=\rho R^{2} K_{\text {ring }} I(\varepsilon)
$$

$$
T=\pi \rho U^{2} D^{2} L / 8=\rho R K_{\text {ring }}^{2} E(\varepsilon)
$$




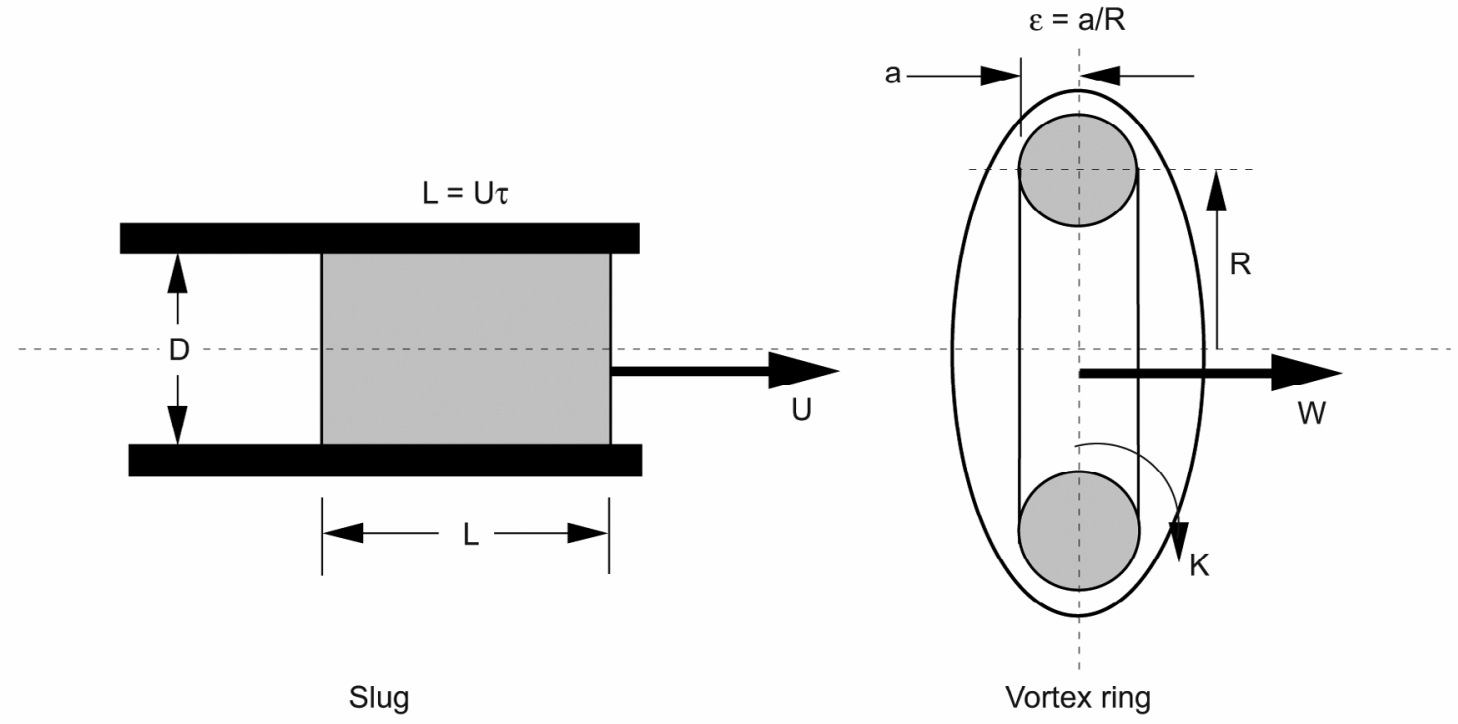

Fig. 1 Schematic diagram of the slug model of vortex ring formation.

In these equations, $I(\varepsilon)$ and $E(\varepsilon)$, the dimensionless values of the impulse and energy, are functions of $\varepsilon$ only. Following Linden and Turner, these equations can be combined to give

$$
\begin{aligned}
(L / D) \Gamma^{1.5} & =\sqrt{\pi / 2\left(P / \rho R^{2} K_{\text {ring }}\right) /\left(T / \rho R K_{\text {ring }}{ }^{2}\right)} \\
& =\sqrt{\pi I(\varepsilon) / 2} / E(\varepsilon)
\end{aligned}
$$

The right hand side of this equation, and hence $L / D$ also, is a function of $\varepsilon$ only. Norbury ${ }^{6}$ solved the equations for a vortex ring numerically, to obtain values of $I(\varepsilon)$ and $E(\varepsilon)$ at a few values of $\varepsilon$. Since the interest here is in small values of $\varepsilon$, at several values of this ratio, it is preferable to have a continuous (in $\varepsilon$ ) representation. Such a representation was found by Fraenkel ${ }^{7}$ who used a solution as a series in $\varepsilon$, valid for small $\varepsilon$. Fraenkel's solutions are

and

$$
I(\varepsilon)=\pi\left(1+3 \varepsilon^{2} / 4\right)
$$

$$
E(\varepsilon)=0.5 \ln (8 / \varepsilon)-7 / 8+\left(3 \varepsilon^{2} / 16\right) \ln (8 / \varepsilon)
$$

However, Fraenkel's values do not agree perfectly with those of Norbury, and so Fraenkel's expressions were modified slightly to give better agreement with Norbury's values of impulse and energy, by using polynomial fits in $\varepsilon$, giving

$$
\begin{aligned}
& I(\varepsilon)=\pi\left(1+0.303 \varepsilon+0.6184 \varepsilon^{2}-0.3563 \varepsilon^{3}\right) \\
& E(\varepsilon)=0.5 \ln (8 / \varepsilon)-0.8594+0.098 \varepsilon^{2} \ln (8 / \varepsilon)
\end{aligned}
$$

Values of $I(\varepsilon)$ and $E(\varepsilon)$ for four values of $\varepsilon$ as given by Norbury, Fraenkel, and the series of equations (7) and (8) are given in table 1 . As can be seen, the series of equations (7) and (8) are good fits to the Norbury values, and will be used below, and in the plots in figure 2 .

In figure 2(a), $L / D$ is plotted against $\varepsilon$ as a red line, using equations (7) and (8) in equation (4b), with $\Gamma=1$, as assumed by Linden and Turner. Also, results from Linden and Turner are shown as star shaped points, for $\varepsilon=0.2$, 0.4 , and 0.6 .

The agreement is quite good, even for $\varepsilon=0.6$. The limiting value of $L / D=4$ is found at a value of $\varepsilon=0.55$, so higher values of $\varepsilon$ than this should not be needed.

The size of the vortex ring is found by combining equations. (1) and (2) leading to an equation for $R / D$ in terms of $I(\varepsilon)$, and hence, through equation $(4 \mathrm{~b}), L / D$. 


$$
R / D=1 / \sqrt{2 \Gamma I(\varepsilon) / \pi}=1 / \sqrt{2 \Gamma\left(1+0.303 \varepsilon+0.6184 \varepsilon^{2}-0.3563 \varepsilon^{3}\right)}
$$

Fraenkel also has an equation for the vortex ring velocity, $W$, namely

$$
W=\left(K_{\text {ring }} / 4 \pi R\right)\left[\ln (8 / \varepsilon)-1 / 4-\varepsilon^{2}\{(3 / 8) \ln (8 / \varepsilon)-15 / 32\}\right]
$$

For $\varepsilon=0$ this agrees with the equation given by Lamb ${ }^{8}$ for small core radius. Again this can be modified to agree with the calculated results of Norbury better by using the fit

$$
W=\left(K_{\text {ring }} / 4 \pi R\right)\left[\ln (8 / \varepsilon)-1 / 4-0.85 \varepsilon^{2}\{(3 / 8) \ln (8 / \varepsilon)-15 / 32\}\right]
$$

Using equations (1) and (9) in equation (11), the ring velocity divided by the slug velocity is seen to be a function of $L / D$ and $\varepsilon$ only,

$$
\begin{aligned}
W / U= & \Gamma^{1.5}(L / D) \sqrt{2\left(1+0.303 \varepsilon+0.6184 \varepsilon^{2}-0.3563 \varepsilon^{3}\right)} \\
& \times\left[\ln (8 / \varepsilon)-1 / 4-0.85 \varepsilon^{2}\{(3 / 8) \ln (8 / \varepsilon)-15 / 32\}\right] / 8 \pi
\end{aligned}
$$

\begin{tabular}{|c|c|c|c|c|c|}
\hline$\varepsilon$ & & 0.2 & 0.4 & 0.6 & 0.8 \\
\hline \multirow[t]{3}{*}{$I(\varepsilon)$} & Norbury & 3.226 & 3.422 & 3.653 & 3.888 \\
\hline & Fraenkel & 3.236 & 3.519 & 3.990 & 4.650 \\
\hline & Fit (Eq. 7) & 3.229 & 3.419 & 3.656 & 3.888 \\
\hline \multirow[t]{3}{*}{$E(\varepsilon)$} & Norbury & 0.9999 & 0.6733 & 0.5264 & 0.4366 \\
\hline & Fraenkel & 0.9971 & 0.7127 & 0.5950 & 0.5526 \\
\hline & Fit (Eq. 8) & 0.9995 & 0.6854 & 0.5271 & 0.4363 \\
\hline \multirow[t]{3}{*}{$W / U$} & Norbury & 0.4363 & 0.5353 & 0.6019 & 0.6522 \\
\hline & Fraenkel & 0.4393 & 0.5188 & 0.5761 & 0.6026 \\
\hline & Fit (Eq. 17) & 0.4381 & 0.5272 & 0.6034 & 0.6516 \\
\hline
\end{tabular}

Table 1. Values of the nondimensional vortex ring impulse $I(\varepsilon)$,energy $E(\varepsilon)$, and velocity $W / U$

and hence can be expressed in terms of $L / D$. The values of $W / U$ from Norbury, Fraenkel, and equation (12) are also given in table 1, again showing good agreement between the Norbury values and the modified fits. $R / D$ from equation (9) (green line) and $W / U$ from equation (12) (blue line), again for $\Gamma=1$, are plotted against $L / D$ in figure 2(a). $W / U$ agrees well with results from Linden and Turner (the diamond shaped points). However, the calculated values of $R / D$ do not agree with experimental values of $R / D$ from Liess and Didden ${ }^{9}$ (the square points) in their dependence on $L / D$. The calculated values decrease with increasing $L / D$, whereas the experimental values increase.

Shariff and Leonard ${ }^{10}$ have plotted ring circulation divided by slug circulation, i.e., $\Gamma$, against $L / D$, using data from Didden, ${ }^{11}$ and Maxworthy, ${ }^{12}$ and find that this ratio is not unity, and decreases with increasing $L / D$. They quote two expressions for $\Gamma$, but neither fits all the data. Both are close at $L / D=1$, with $\Gamma=1.4$, which does give the experimental value of $R / D$ found by Liess and Didden. By finding a value of $\Gamma$ that also fits the Liess and Didden value of $R / D$ at $L / D=2.5$, the constants in the following expression were found,

$$
\Gamma=K_{\text {ring }} / K_{\text {slug }}=0.66+0.74 /(L / D)
$$

This agrees with the two expressions quoted by Shariff and Leonard at $L / D=1$, and falls between them at $L / D=2.5$, so it does not seem unreasonable. Admittedly, Shariff and Leonard point out that the reason for the discrepancy between the two expressions may be due to a Reynolds Number dependence. This will be ignored here in the interests of simplicity. Using $\Gamma$ from equation (13), $\varepsilon, R / D$, and $W / U$ were calculated as functions of $L / D$, and are plotted in figure 2(b). Now there is excellent agreement between the calculated values of $R / D$ and the experimental values of Liess and Didden. 

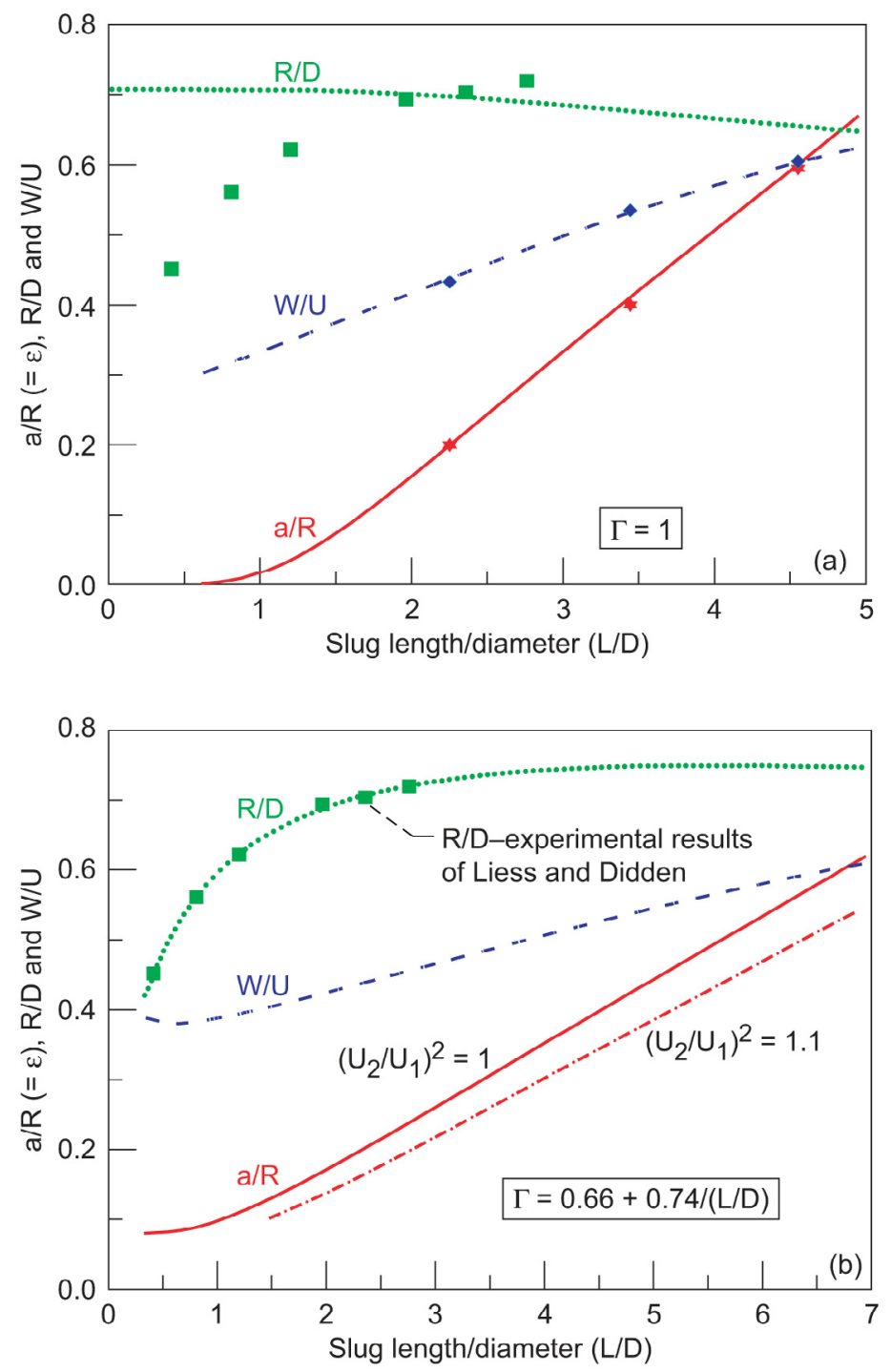

Fig. 2 (a) Calculations of $\varepsilon, R / D$ and W/U against $L / D$, using the slug model with $\Gamma=1$, and, (b) with $\Gamma=0.66+0.74 /(\mathrm{L} / \mathrm{D})$. The calculated $\varepsilon$ is from equation (4b) with equations (7) and (8), plotted as the solid line $(-)$ for $\left(\mathrm{U}_{2} / \mathrm{U}_{1}\right)^{2}=1$, and as a dot-dash line $(-\cdot-\cdot--)$ for $\left(U_{2} / U_{1}\right)^{2}=1.1$, W/U is from equation (12), plotted as a dash line (- - - ), and R/D is from equation 12 , plotted as a dotted line (.........). Also plotted are calculated results from Linden and Turner ${ }^{5}$ for $\varepsilon(*)$, and W/U $(\bullet)$, and experimental values of R/D from Liess and $\operatorname{Didden}^{9}(\square)$.

It was implicitly assumed above that $U$ was a constant independent of time. This is rarely true in practice. If the actual temporal distribution of velocity from the source is represented by $u(t)$ then it follows that $L=\int_{0}^{\tau} u(t) d t$, and the jet circulation, impulse and energy are

$$
K_{\text {slug }}=\int_{o}^{\tau} u(t)^{2} d t / 2=U_{1} L / 2
$$




$$
\begin{aligned}
& P=\pi \rho D^{2} L / 4\left(\int_{o}^{\tau} u(t)^{2} d t / \int_{o}^{\tau} u(t) d t\right)=\pi \rho D^{2} L U_{1} / 4 \\
& T=\pi \rho D^{2} L / 8\left(\int_{o}^{\tau} u(t)^{3} d t / \int_{o}^{\tau} u(t) d t\right)=\pi \rho D^{2} L U_{2} 2 / 8
\end{aligned}
$$

where the integrals inside the parentheses define $U_{I}$ and $U_{2}$, and are taken over one pulse. The right hand side of equation (4) is now multiplied by $\left(U_{2} / U_{1}\right)^{2}$. The effect on the curve of $\varepsilon$ versus $L / D$ of having a time dependant flow is shown in figure $2(\mathrm{~b})$, for a case with $\left(U_{2} / U_{1}\right)^{2}=1.1$.

In summary, given the slug velocity, including its temporal distribution, length and diameter, the vortex ring circulation, radius, and core radius, and hence velocity, can all be determined, but it is essential to input $\Gamma$ as a function of $L / D$. Whether the circulation for an annular jet can be derived from equation (1) will be determined later.

\section{Experimental Apparatus}

\section{A. Pulsed Jet Source}

The Hartmann-Sprenger, or resonance, tube consists of a steady, sonic or supersonic jet, which is flowing into a closed tube. Under certain conditions, a periodic cycle is established in which the jet first fills the tube, then a hammer shock inside the tube empties the tube, deflecting the jet from the tube in the process. When the tube pressure has fallen sufficiently, the cycle can begin again. What was not known at the start of this effort was whether this phenomenon could also produce a directed, pulsed jet. To attempt this, a cylindrical shroud was placed around the tube and jet, to collect the air leaving the tube and direct it out the back of the shroud. Hartmann-Sprenger tubes have been shrouded previously (Brocher and Pinna $^{13}$ ), but with acoustic horns, with the objective of amplifying the sound. These acoustic horns were closed at the end where the source is located, and increased in area with distance away from the source. The flow from the horn would therefore be diverging, and the velocity at the exit would be reduced in value from that leaving the source. In order to create a more concentrated flow, a cylindrical shroud was used in the present work. This shrouded tube did produce vortex rings traveling along the extended axis of the device. The frequency of operation is dependant primarily on the length of the closed tube, but also on the length of the shroud.

The shrouded tube used at a frequency of $550 \mathrm{~Hz}$ is shown in figure 3. A Mach 2 axisymmetric nozzle with a $12.7 \mathrm{~mm}$ diameter throat was aligned with a resonance tube $152 \mathrm{~mm}$ in length, internal diameter $16.8 \mathrm{~mm}$, and external

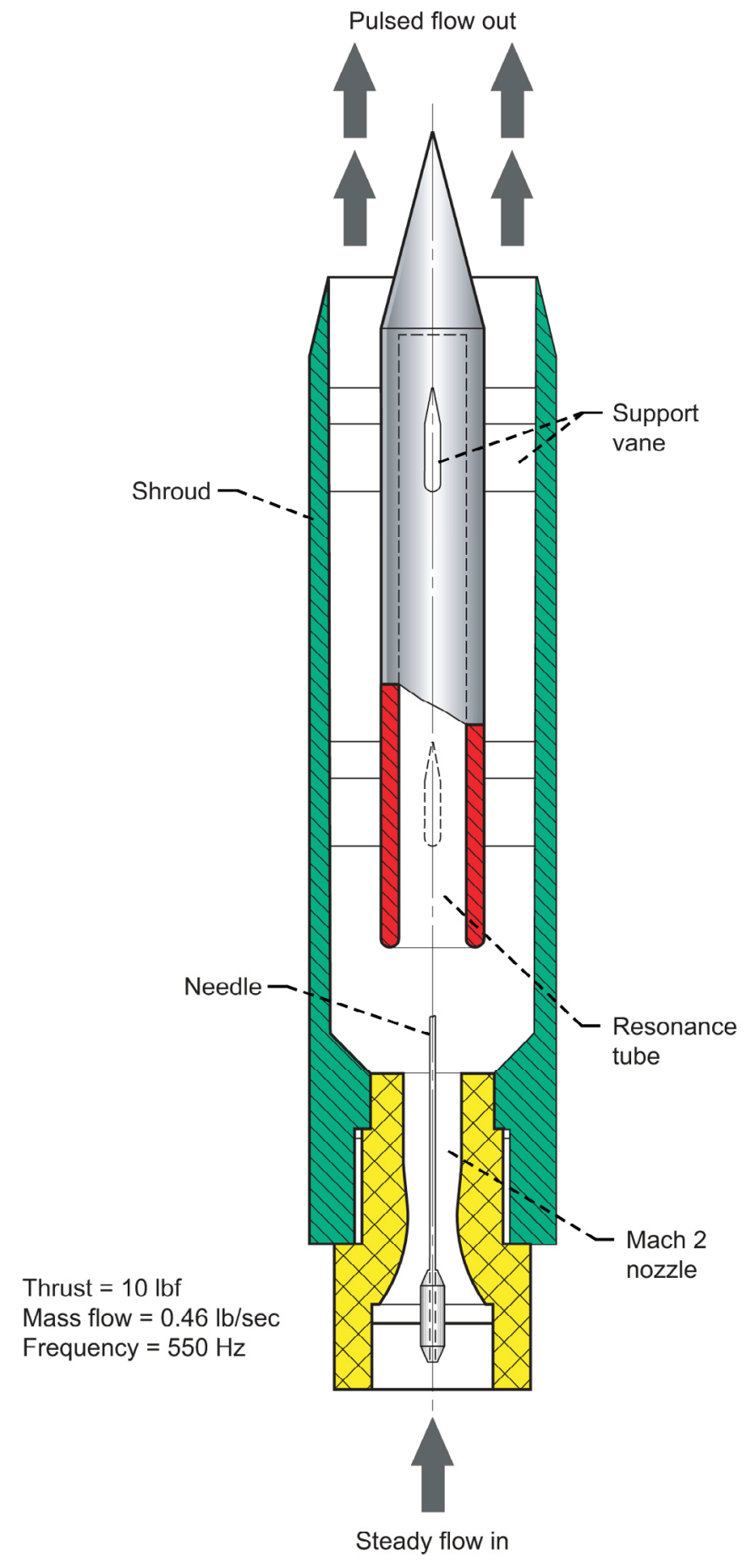

Fig. 3 Schematic drawing of the shrouded Hartmann-Sprenger tube. 
diameter, $d_{t}=25.4 \mathrm{~mm}$. This was surrounded by a shroud, of internal diameter $D_{\mathrm{s}}=50.8 \mathrm{~mm}$. A needle was aligned with the axis of the jet to stimulate oscillations, as demonstrated by Brocher. ${ }^{14}$ Use of the needle makes the device relatively insensitive to the distance between nozzle exit and the tube entrance. A supply of air at a pressure of 7.8 atmospheres ensured Mach 2 operation in the nozzle when exhausting directly to the atmosphere. The average mass flow was measured upstream of the nozzle, using an orifice. To generate a frequency of $1100 \mathrm{~Hz}$, a plug was inserted into the resonance tube, effectively shortening it to $76 \mathrm{~mm}$ in length. New tubes and shrouds were built for frequencies of 275 and $125 \mathrm{~Hz}$.

\section{B. Measurements}

A thrust plate, $762 \mathrm{~mm}$ in diameter, was used to measure the average thrust produced by the pulsating jet. The thrust plate was attached to an Omega load cell model LC601-25, having a range of \pm 111 Newtons, to provide an electrical thrust signal. The signal from the load cell was fed to an Agilent model 34401A averaging multimeter. All runs lasted one minute, during which time the voltmeter stored 180 readings, and then displayed the average value. The experimental procedure involved making six tests to read the thrust of the jet. The jet thrust, $T_{j e t}$, defined as the average of the six test readings, typically measured $39.4 \pm 0.5$ Newtons.

In order to measure the properties of the vortex rings produced, namely velocity, radius and core radius, the rings were sampled with a pair of fast response transducers (Endevco model 8530C-50), each built into the nose of hemisphere-cylinder body, and separated by a distance of $25.4 \mathrm{~mm}$. The probes were inserted into the flow $76 \mathrm{~mm}$ downstream of the jet exit. The signals from the probes were displayed on an oscilloscope, using dc coupling. The pair was moved radially to generate a plot of pulse pressure versus radius for the vortex rings. This information gave an indication of the vortex radius and core radius. Then, by spacing the probes equidistantly from the jet axis, at a radius at which the probes gave the minimum pressure signals (the minima being sharper than the maxima), but separating the probes axially, a measurement of the vortex ring velocity could be made. This was repeated for each frequency. For the $275 \mathrm{~Hz}$ frequency only, a laser particle image velocimeter (LPIV), as described by John, Paxson, and Wernet, ${ }^{15}$ was used to give a velocity and vorticity map of the vortex ring. One of the high frequency pressure probes was also placed at the jet exit to obtain the jet exit velocity as a function of time.

\section{A. Vortex Ring Probing}

\section{Experimental Results}

Signals from the high frequency pressure probes sampling the vortex rings at a distance (usually $76 \mathrm{~mm}$ ) from the nozzle exit, are shown in the oscillogram in figure 4(a), taken at a frequency of $550 \mathrm{~Hz}$, As indicated in the sketch in figure 4(b), two probes were used, both aligned with the $z$ axis, and spaced $25.4 \mathrm{~mm}$ apart. The upper oscillogram is from the probe closer to the axis of the jet, and exhibits a rise in pressure with time, followed by a decay, returning to atmospheric pressure at about half the period of the pulses. After the signal returned to atmospheric, there appeared to be a high frequency oscillation on it, lasting until the next pulse. As the probe was moved radially, away from the jet axis, the value of the peak pressure first increased slightly, then decreased to zero at a radial position equal to about 1.2 times the jet radius. At distances greater than this, a pulse was seen which was a decrease in pressure, with a minimum pressure well below atmospheric, as shown by the lower oscillogram in figure 4(a), which is from the probe further from the axis. The values of the pulse extrema, expressed as pressures, are plotted as a function of distance from the jet axis in figure 4(b). For a frequency of $550 \mathrm{~Hz}$, the absolute outer edge of the disturbance, where there is no longer any signal, is at a radial position of 3.2 times the jet radius, i.e., $81 \mathrm{~mm}$. These measurements were repeated at each frequency used.

The positive signals are generated when the probe is acting as a pitot probe, with flow directed towards the transducer. The negative signals are partly generated when the probe is in the core of the vortex ring, such that the flow is in the opposite direction, and the probe is acting more like a static pressure probe. This will become more obvious below.

As stated above, in order to measure the vortex ring velocity $W$, the probes were separated axially by a distance varying between 50 to $100 \mathrm{~mm}$, depending on the frequency, and were each at a radial distance from the axis such that they were sampling the pressure minima. Velocities derived from these signals are given in table 2. Each velocity is an average of about 15 readings. Some variation in velocity with probe axial separation, and with position of the leading probe relative to the nozzle exit, was observed, but was less than the experimental error. Also listed in table 2 are the values of the thrust of the jet $T_{j e t}$ alone for each frequency. The thrust was not the same at each frequency used, although the mass flow, $\dot{m}_{j e t}$, was. This implies that the jet exit velocity $U$ was not the same for each frequency. Since the vortex ring velocity is proportional to $U$, it is appropriate for comparison to correct the vortex ring velocity to a common thrust of 39.4 Newtons by multiplying the observed value of $W$ by $39.4 / T_{j e t}$. The corrected values are also given in table 2 . 

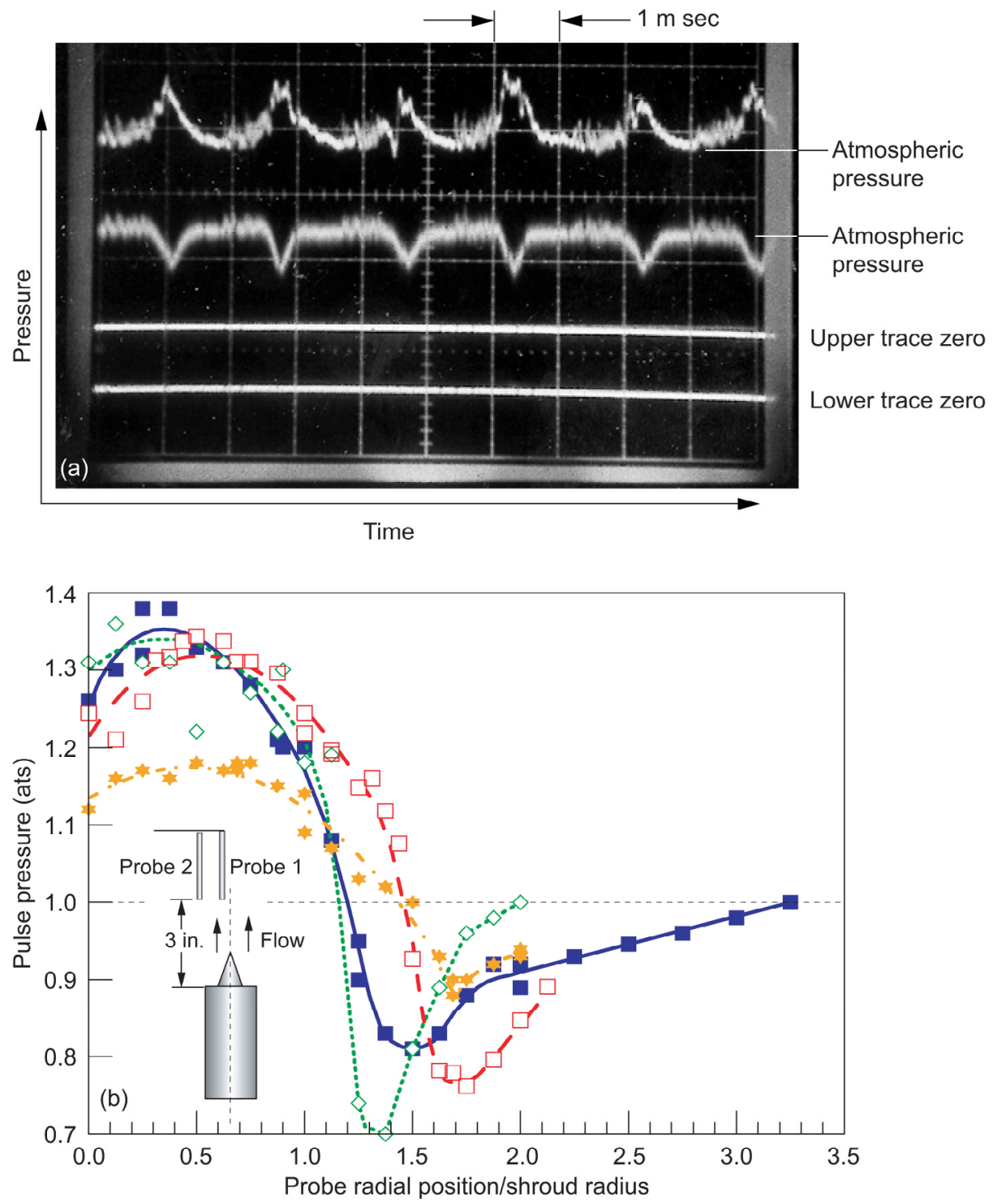

Fig. 4 (a) Oscillograms of high frequency pressure probe data; (b) plot of the pressure pulse extrema versus normalized probe position; $(\diamond)$, data for $f=1100 \mathrm{~Hz} ;(\bullet \cdots \cdots . \bullet)$, fit to data for $\mathrm{f}=1100 \mathrm{~Hz} ;(\square)$, data for $\mathrm{f}=550 \mathrm{~Hz}$; ( $(\longrightarrow)$, fit to data for $\mathrm{f}=550 \mathrm{~Hz} ;(\square)$, data for $f=275 \mathrm{~Hz} ;(----)$, fit to data for $f=275 \mathrm{~Hz}$; ( $)$, data for $f=125 \mathrm{~Hz} ;(--\cdot--)$, fit to data for $f=125 \mathrm{~Hz}$.

Table 2. Measured vortex ring velocity, $W$, and thrust corrected vortex ring velocity

$$
\mathrm{f}=1100 \mathrm{~Hz} \quad \mathrm{f}=550 \mathrm{~Hz} \quad \mathrm{f}=275 \mathrm{~Hz} \quad \mathrm{f}=125 \mathrm{~Hz}
$$

\begin{tabular}{lllll} 
W from Pitot Probes (m/s) & 73 & 77 & 83 & 77 \\
W from LPIV & & & 79 & \\
Thrust (Newtons) & 40.0 & 44.7 & 39.3 & 33.8 \\
W corrected to 39.4 Newtons & 72 & 68 & $83 / 79$ & 89 \\
\hline
\end{tabular}

\section{B. Laser Particle Imaging Velocimetry}

Laser particle image velocimetry was used to measure velocities within the vortex ring, for a frequency of $275 \mathrm{~Hz}$ only. The values of axial velocity along a radius at the centre of the ring are plotted in figure 5. The velocity of the vortex ring itself is $79 \mathrm{~m} / \mathrm{s}$. This velocity occurs at the vortex radius $R$. The maxima and minima of velocity 
are at radii of $R-a$, and $R+a$ respectively, from which $R=33.3 \mathrm{~mm}, a=18 \mathrm{~mm}$, and hence $\varepsilon=0.54$. A sketch of the vortex ring is on the right hand side of figure 5. The minimum signal from the pressure probes is at a radius of $43.2 \mathrm{~mm}$, at which radius the velocity is zero. This radius is close to $R+a / 2$. For greater radii, the velocity over the probes is negative, so they are not then acting as total pressure probes.

The LPIV data provide a map of vorticity $\omega$ over the entire field of view. The vorticity along the vortex radius is also shown in figure 5. The vorticity is zero at a large distance from the vortex, then after a region of small negative vorticity, increases as the distance decreases, peaks, then decreases to zero at a radius of $15.8 \mathrm{~mm}$, and goes to a negative peak before returning to zero on the axis. The positive peak of vorticity occurs at the vortex ring radius, which is also the radius at which the velocity is equal to the vortex ring velocity. The circulation in the vortex ring can be determined from the vorticity by performing the integral

$$
K_{\text {ring }}=\iint \omega d r d z
$$

over the whole flow field. The result is $K_{\text {ring }}=13.7 \mathrm{~m}^{2} / \mathrm{s}$. The integration was performed numerically from large radius to zero. The circulation increased as the radius decreased, reaching a maximum of $23.9 \mathrm{~m} \mathrm{~m}^{2} / \mathrm{s}^{-1}$ at a radius of $15.8 \mathrm{~mm}$, then decreasing to the value of $13.7 \mathrm{~m}^{2} / \mathrm{s}^{-1}$ on the centerline. This is a consequence of the flow coming from an annulus, so that vorticity shed from the inner diameter is of opposite sign from that generated at the outer diameter, thereby reducing the total circulation. However the negative vorticity shed at the inner diameter does not cancel that shed at the outer diameter.

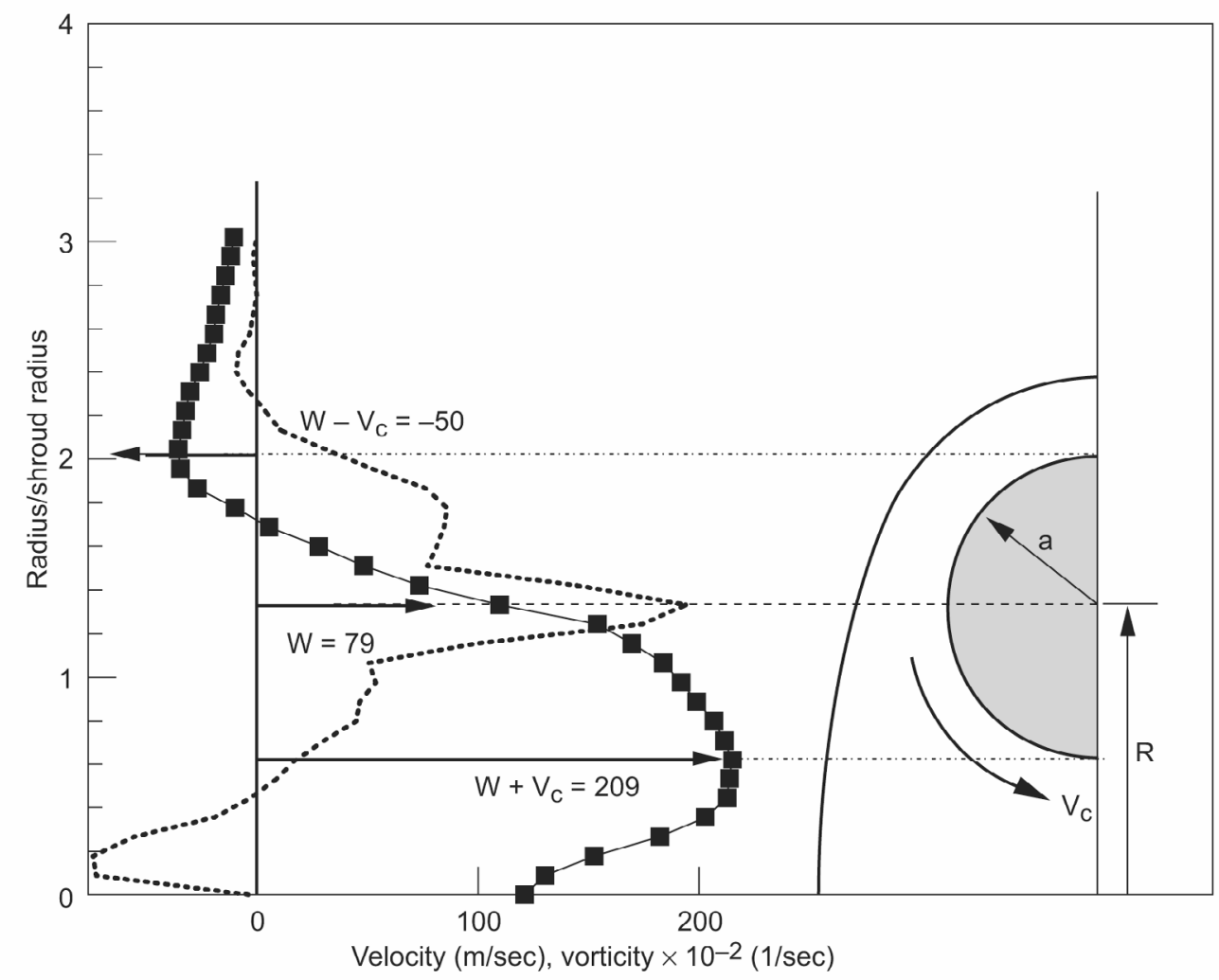

Fig. 5 Velocities (- - ) on the $r$-axis through the vortex ring as a function of normalized radial position. The velocities come from LPIV data. $V_{C}=K / 2 \pi a=130 \mathrm{~m} / \mathrm{s}$ is the velocity at the edge of the core. The vorticity (........) along the $r$-axis divided by 100 is also plotted. A sketch of the vortex ring is given at the right. 


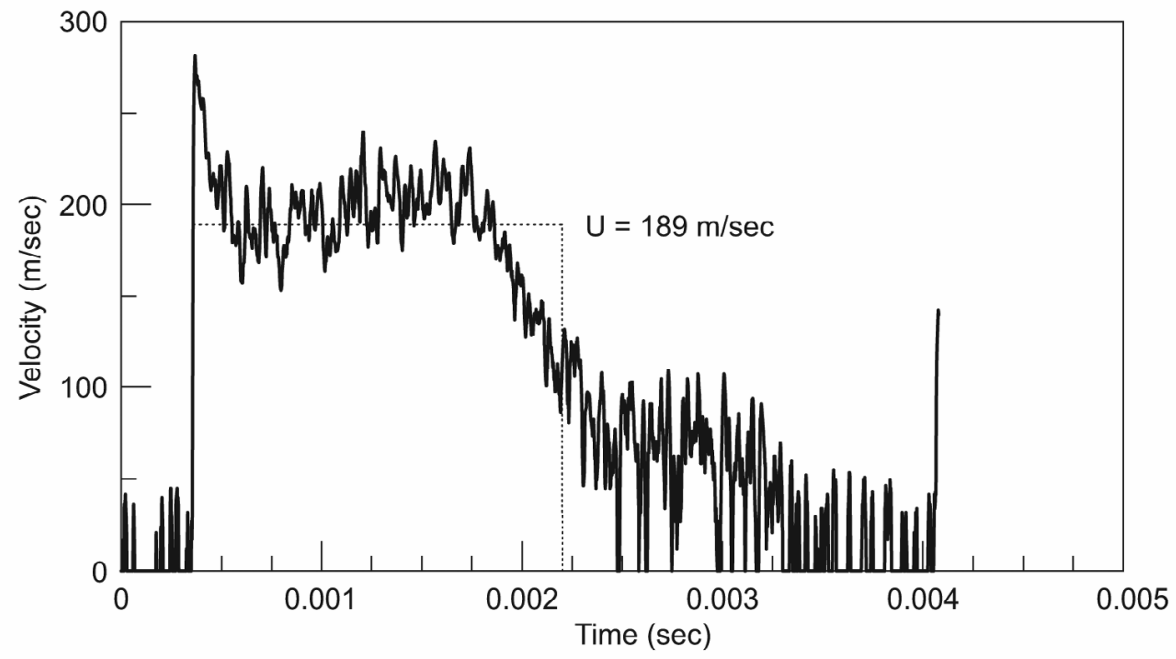

Fig. 6 Flow velocity at the exit of the Hartmann-Sprenger tube as a function of time, for $f=275 \mathrm{~Hz}$. Data from the high frequency pressure probe.

\section{Jet Exit Velocity}

By placing one of the high frequency pressure probes used to sample the vortex ring exactly in the exit plane of the jet, a signal is obtained which can be related to the jet exit velocity. This was done at a frequency of $275 \mathrm{~Hz}$ only. The resulting velocity is plotted in figure 6 . The flow starts with a weak shock wave (Mach number $=1.25$ ), and, after an initial spike, is approximately constant for a while before falling off. An average flow exit velocity can be derived from the thrust, namely

$$
U=T_{j e t} / \dot{m}_{j e t}
$$

this gives a value of $189 \mathrm{~m} / \mathrm{sec}$, which is also shown in figure 6 , and is in good agreement with the probe results. The probe velocities can be integrated to give a value for $\left(U_{2} / U_{1}\right)^{2}$ of 1.1, the value used in figure 2(b) in deriving $L / D$ versus $\varepsilon$ for a jet with velocity that is not constant with time.

\section{Comparison between the Slug Model and Experimental Results}

The experiments were performed at four different frequencies. Since the mass flow was constant at all frequencies, the mass per pulse $m_{p}$ is given by

$$
m_{p}=\dot{m}_{j e t} / f
$$

in which $f$ is frequency. But $m_{p}$ is also given by the product of pulse volume and density

$$
m_{p}=\rho \pi\left(D_{s}^{2}-d_{t}^{2}\right) L / 4
$$

from which it is seen that $L / D$ is inversely proportional to frequency. In referring to the slug flow model, it is clear that the appropriate diameter appearing in $L / D$ is twice the square root of the flow area divided by $\pi$, so that for the Hartmann-Sprenger tube it is $D=\sqrt{\left(D_{s}^{2}-d_{t}^{2}\right)}$. In table 3 , the values of $L / D$ for each frequency used are given together with values of $\varepsilon$ from equation (4) for the two higher frequencies. For the lower frequencies, since the vortex probing showed that the vortex rings were the same size for both frequencies, $\varepsilon$ is the same for both, and equal to the value measured from the LPIV data, namely 0.54 . Gharib et al. ${ }^{4}$ showed that above some value of $L / D$, called the formation number, the circulation of the vortex ring remains constant as $L / D$ increases, with the remaining circulation going into a trailing jet. In the case of Gharib et al., the limiting value of $L / D$ was 4 , consistent with a jet velocity constant in time at $\varepsilon=0.55$. If this were the case for the present experiments, the vortex rings for 550, 275, and $125 \mathrm{~Hz}$, which all have $L / D$ greater than 4 , should be the same size. In fact the $550 \mathrm{~Hz}$ vortex rings are smaller 
Table 3. $L / D$ and $\varepsilon$ for each frequency used

\begin{tabular}{|c|c|c|c|c|}
\hline Frequency $(\mathrm{Hz})$ & 1100 & 550 & 275 & 125 \\
\hline$L / D$ & 2.5 & 5 & 10 & 22 \\
\hline$\varepsilon$ & 0.177 & 0.386 & 0.54 & 0.54 \\
\hline
\end{tabular}

than those at 275 and $125 \mathrm{~Hz}$. This indicates that the formation number appears at a higher value of $L / D$ than 4 . From equation (4), with $\left(U_{2} / U_{1}\right)^{2}=1.1$, the maximum value of $\varepsilon$ observed, i.e., 0.54 , occurs at an $L / D$ of 6.86 . This will then be taken to be the formation number $N$, for these vortex rings. Although the value of $N$ is different from the value of Gharib et al., ${ }^{4}$ the value of $\varepsilon$ at the formation number appears to be the same, suggesting that it is $\varepsilon$ that has a limiting value rather than $L / D$.

The probe measurements in figure 4(b) show that the pulse minima are at a value of $r / R_{s}=1.7$ for the $275 \mathrm{~Hz}$, and $125 \mathrm{~Hz}$ rings, where $r$ is radius, and $R_{s}$ the shroud radius. The LPIV data in figure 5 indicate that this is the radius at which the velocity is zero, and is approximately equal to $R+a / 2$. Assuming then that the probe minima are at a radius of $R+a / 2$, and using the values of $\varepsilon$ in table 3, the value of $R+a$ can be calculated for each frequency, and plotted against $L / D$. The results are shown in figure 7, together with the LPIV value at $275 \mathrm{~Hz}$, and the calculated values from the slug model, assuming a formation number of 6.86 . The agreement is very good.

In figure 8 , the observed vortex ring velocities, as corrected for thrust, are also plotted against $L / D$, together with the values calculated from the slug model, again assuming a formation number of 6.86 . Two calculated values are given, one for a value of $K_{\text {ring }}=13.7 \mathrm{~m}^{2} / \mathrm{s}$, corresponding to the overall circulation of the vortex ring from the LPIV data, and one for $K_{\text {ring }}=20 \mathrm{~m}^{2} / \mathrm{s}$, corresponding to the value from $K_{\text {ring }}=\Gamma U L / 2$, i.e., equation (1), with $\Gamma=0.768$ at $\varepsilon=0.54$. Even though the data show some scatter, it is clear that the velocity calculated with $K_{\text {ring }}=20 \mathrm{~m}^{2} / \mathrm{s}$ is considerably greater than the observed values. It might be thought that the high value of $W$ calculated from the slug theory is because the value of $\Gamma$ used in equation (1) is too high. However, $\Gamma$ also appears in equation (9), the equation for the vortex ring radius, so if $\Gamma$ were reduced, the good agreement between calculated and observed ring radius would be diminished. In fact the discrepancy is because the circulation calculated using equation (1) is based on the circulation created at the outer edge of the shroud only. As shown by the LPIV data, there is vorticity of opposite sign shed at the inner tube, which reduces the total circulation. Using the circulation derived from the LPIV data, the calculated vortex ring velocities are in much better agreement with the data.

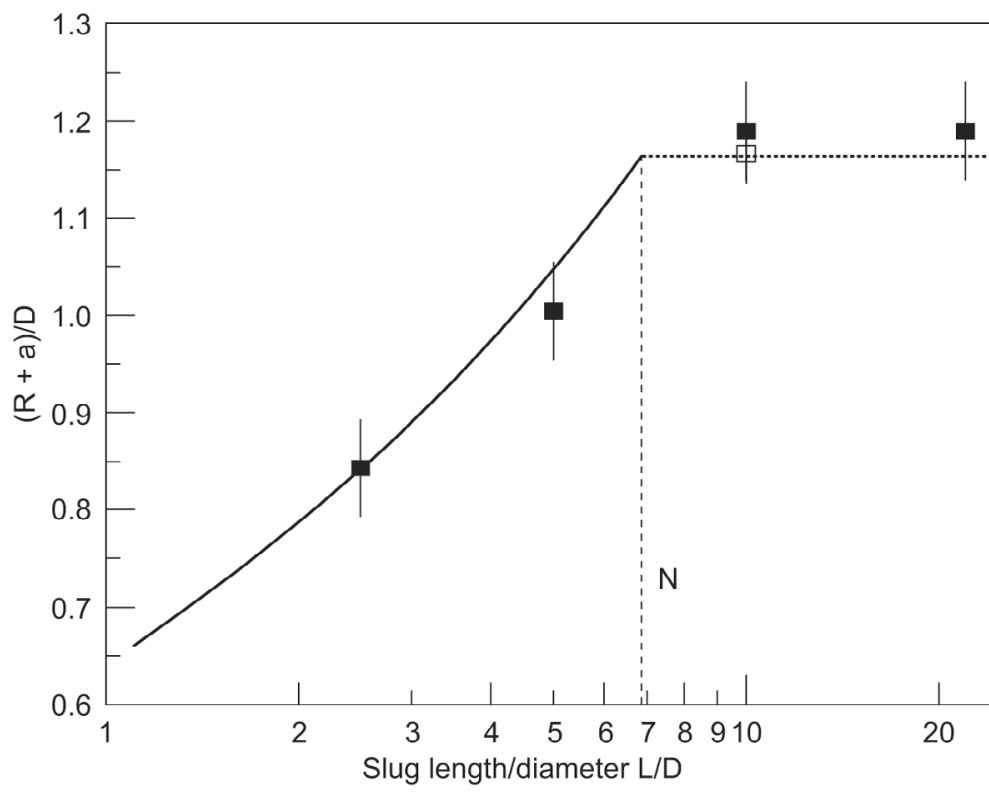

Fig. $7(R+a) / D$ against $L / D$, with $(R+a) / D$ calculated from the slug model (-), an extension at constant value for L/D greater than $\mathrm{N}(----)$, and data from pressure probes $(\boldsymbol{\bullet})$, and from LPIV measurements (). 


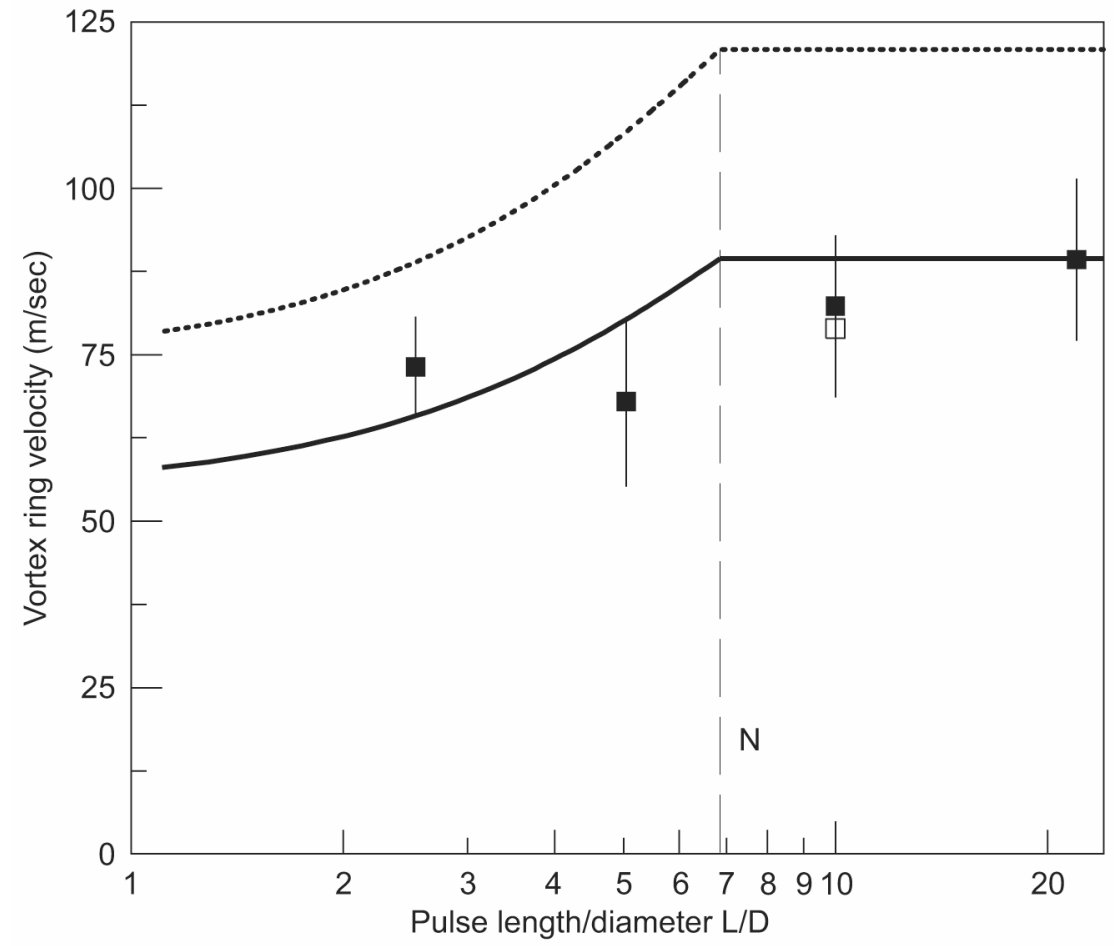

Fig. 8 Vortex ring velocity plotted against L/D; as calculated from the slug model with $\mathrm{K}_{\text {ring }}=13.7 \mathrm{~m}^{2} / \mathrm{s}(\longrightarrow)$, as calculated from the slug model with $\mathrm{K}_{\text {ring }}=20 \mathrm{~m}^{2} / \mathrm{s}(-\mathbf{- -})$, together with data from pressure probes $(\mathbf{\square})$, and data from LPIV ().

\section{Comments}

If the flow in the jet as it leaves the shroud is approximately uniform radially, there should be approximately equal and opposite circulation generated at the shroud and the inner tube in the jet flow. However, as pointed out by Maxworthy, ${ }^{12}$ the internal flow in the case of a circular jet is not the only contributor to the circulation, a negative circulation appears in the induced flow external to the jet, partially canceling that due to the jet. For the annulus, it is not clear what is the boundary condition between jet flow at the inner diameter of the jet and the flow centerline. There is probably a trapped region of separated flow, creating a complex flow pattern. It is interesting that the peak of the negative vorticity is much closer to the centerline than the outer radius of the inner tube. It is also possible in the present flow that some circulation exists in the jet before it leaves the shroud. The present measurement of velocity did not have sufficient radial resolution to examine this possibility. Certainly the LPIV results show a definite net overall circulation, but the exact value would appear to be a field for further investigation.

\section{Conclusions}

In conclusion, the flow from the shrouded Hartmann-Sprenger tube has been shown to be a train of vortex rings. The vortex ring size and velocity have been measured using probes and LPIV, with good agreement between the two techniques. From the leveling-off of both $(R+a)$ and velocity at high $L / D$, the formation number was found to be 6.9 , at a value of $\varepsilon=0.54$. The slug model predicts the vortex ring size correctly, provided proper account is taken of the dependence of the ratio of ring circulation to slug circulation on $L / D$, and of the temporal velocity distribution in the jet in calculating the relationship between $\varepsilon$ and $L / D$. The vortex ring velocity is proportional to the vortex ring circulation, which is not given correctly for an annulus by the equation $K_{\text {ring }}=\Gamma U L / 2$ as this ignores the negative circulation generated at the inner radius. However, vortex ring velocities calculated with the circulation derived from the LPIV measurements were consistent with the experimentally measured vortex ring velocities. When the time dependence of the flow is accounted for, the slug model gives a value of $N$ that is also consistent with the experiment, for cut-off at $\varepsilon=0.54$. 


\section{References}

${ }^{1}$ Wilson, J., and Paxson, D.E. "Unsteady Ejector Performance: An Experimental Investigation using a Resonance Tube Driver," Paper AIAA-2002-3632, presented at the 38th Joint AIAA/ASME/SAE/ASEE Propulsion Conference, Indianapolis, IN., 2002.

${ }^{2}$ Elder, F.K. and de Haas, N. "Experimental Study of the Formation of a Vortex Ring at the Open End of a Cylindrical Shock Tube," Journal of Applied Physics, 23, pp. 1065-1069, 1952.

${ }^{3}$ Das, D., Arakeri, J.H., Krothapalli, A., and Lourenco, L.M. "Compressible Vortex Ring: A PIV Study" Paper AIAA 20012214, presented at the 7th AIAA/CEAS Aeroacoustics Conference, Maastrich, Netherlands, 2001.

${ }^{4}$ Gharib, M., Rambod, E., and Shariff, K. "A universal time scale for vortex ring formation," J. Fluid Mech., 360, pp. 121-140, 1998.

${ }^{5}$ Linden, P.F., and Turner, J.S. "The formation of 'optimal' vortex rings, and the efficiency of propulsion devices," J. Fluid Mech., 427, pp. 61-72, 2001.

${ }^{6}$ Norbury, J. “A family of steady vortex rings,” J. Fluid Mech., 57, pp. 417-431, 1973.

${ }^{7}$ Fraenkel, L.E. "Examples of steady vortex rings of small cross-section in an ideal fluid,” J. Fluid Mech., 51, pp. 119-135, 1972.

${ }^{8}$ Lamb, Sir H. "Hydrodynamics," Dover, New York, 1932.

${ }^{9}$ Liess, C., and Didden, N. "Experimentelle Untersuchung von Ringwirbeln," 50 Jahre M.P.I. Stroemungsforschung, Goettingen, 1975.

${ }^{10}$ Shariff, K., and Leonard, A. "Vortex Rings," Annu. Rev. Fluid Mech., 24, pp. 235-279, 1992.

${ }^{11}$ Didden, N. "On the formation of vortex rings: Rolling-up and production of circulation," J. Appl. Mech. Phys. (ZAMP) 30: pp. 101-116, 1979.

${ }^{12}$ Maxworthy, T. "Some experimental studies of vortex rings,” J. Fluid Mech. 81, pp. 465-495, 1977.

${ }^{13}$ Brocher, E. and Pinna, G. "Aeroacoustical Phenomena in a Horn Excited by a Hartmann-Sprenger Tube," Acustica, Vol. 45, No. 3, pp. 180-189, 1980.

${ }^{14}$ Brocher, E. "Contribution à l'étude des générateurs acoustique à jet d'air,” Acustica, Vol. 32, pp. 227-235, 1975.

${ }^{15}$ John, W.T., Paxson, D.E., and Wernet, M.P. "Conditionally Sampled Pulsejet Driven Ejector Flow Field Using DPIV," AIAA Paper 2002-3231, 2002.

${ }^{16}$ Paxson, D.E., Wilson, J., and Dougherty, K.T. "Unsteady Ejector Performance: An Experimental Investigation Using a Pulsejet Driver," Paper AIAA-2002-3915, presented at the 38th Joint AIAA/ASME/SAE/ASEE Propulsion Conference, Indianapolis, IN, 2002. 


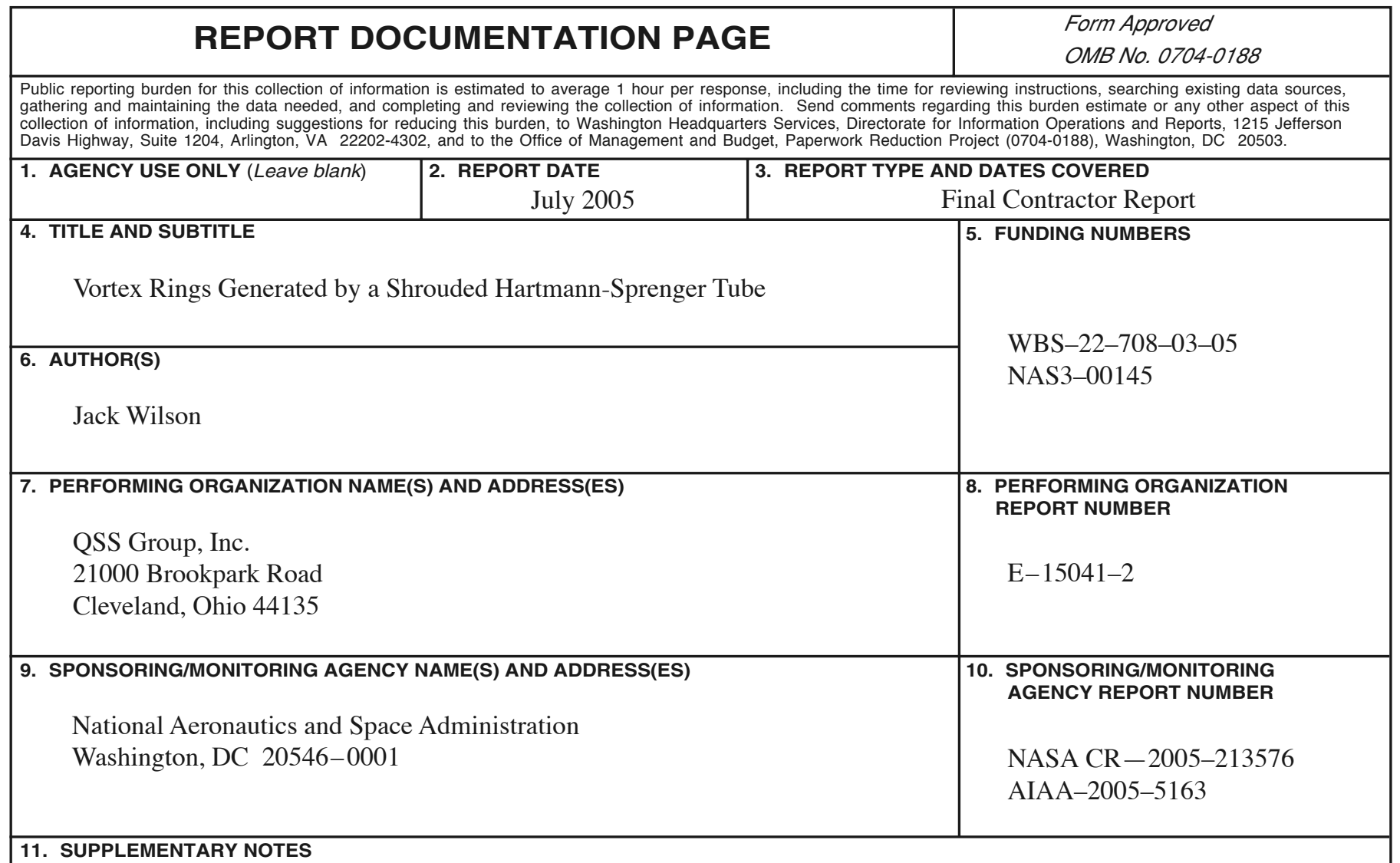

Prepared for the 35th Fluid Dynamics Conference and Exhibit sponsored by the American Institute of Aeronautics and Astronautics, Toronto, Ontario, Canada, June 6-9, 2005. Project Manager, Richard L. DeLoof, Aeronautics Division, organization code PRV, 216-433-6632.

12a. DISTRIBUTION/AVAILABILITY STATEMENT

12b. DISTRIBUTION CODE

Unclassified - Unlimited

Subject Category: 02

Available electronically at http://gltrs.grc.nasa.gov

This publication is available from the NASA Center for AeroSpace Information, 301-621-0390.

13. ABSTRACT (Maximum 200 words)

The pulsed flow emitted from a shrouded Hartmann-Sprenger tube was sampled with high-frequency pressure transducers and with laser particle imaging velocimetry, and found to consist of a train of vortices. Thrust and mass flow were also monitored using a thrust plate and orifice, respectively. The tube and shroud lengths were altered to give four different operating frequencies. From the data, the radius, velocity, and circulation of the vortex rings was obtained. Each frequency corresponded to a different length to diameter ratio of the pulse of air leaving the driver shroud. Two of the frequencies had length to diameter ratios below the formation number, and two above. The formation number is the value of length to diameter ratio below which the pulse converts to a vortex ring only, and above which the pulse becomes a vortex ring plus a trailing jet. A modified version of the slug model of vortex ring formation was used to compare the observations with calculated values. Because the flow exit area is an annulus, vorticity is shed at both the inner and outer edge of the jet. This results in a reduced circulation compared with the value calculated from slug theory accounting only for the outer edge. If the value of circulation obtained from laser particle imaging velocimetry is used in the slug model calculation of vortex ring velocity, the agreement is quite good. The vortex ring radius, which does not depend on the circulation, agrees well with predictions from the slug model.

\begin{tabular}{|c|c|c|c|}
\hline 14. SUBJECT TERMS & 14. SUBJECT TERMS & & $\begin{array}{c}\text { 15. NUMBER OF PAGES } \\
19\end{array}$ \\
\hline NSN 7540-01-280-5500 & & & $\begin{array}{l}\text { andard Form } 298 \text { (Rev. 2-89) } \\
\text { scribed by ANSI Std. Z39-18 } \\
3-102\end{array}$ \\
\hline
\end{tabular}



